NOTICE: This is the author's version of a work accepted for publication by Springer. The final publication is available at link.springer.com:

https://link.springer.com/article/10.1007/s13218-017-0498-4 


\title{
Smart Environments: What is it and why should we care?
}

\author{
Diedrich Wolter • Alexandra Kirsch
}

Received: date / Accepted: date

\begin{abstract}
This article portrays the area of smart environments from a research perspective, defining the field and discriminating it against related fields. We position the field of smart environments within the disciplines of artificial intelligence and human-computer interaction and discuss its genuine research questions. As an application area, smart environments presents prospects on improving our everyday lives, but systems penetrating our environments also trigger crucial questions related to sociology, ethics, and privacy on an unprecedented level.
\end{abstract}

\section{Why smart environments?}

Smart environments is a field attracting researchers from manifold areas of computer science. When trying to explain how we have arrived at today's exiting stateof-the-art in the field, two reasons stand out. First, the technological basis has been laid out. Since G. E. Moore posed his hypothesis on rapidly increasing microchip complexity in the late 1960ies [21] we observe its fulfilment in the availability of increasingly powerful yet more and more cost-effective microprocessors and computing systems. Many appliances are nowadays equipped with remarkable computing power, possibly just because it proves to be the most economical way

\section{Wolter}

Faculty of Information Systems and Applied Computer Sciences

University of Bamberg

E-mail: diedrich.wolter@uni-bamberg.de

\author{
A. Kirsch \\ Department of Computer Science \\ Eberhard Karls Universität Tübingen \\ E-mail: alexandra.kirsch@uni-tuebingen.de
}

of implementation. In the era of internet technology it seems thus only natural to interconnect appliances and devise extra functionality enabled by the availability of a computing platform. The desire to explore and fill this space of possibilities - ideally beyond gadgetism - was complemented by a society that has to face the enormous challenge of an ageing society. The second rationale of smart environments research lies thus in the prospects of appliances and environments that assist their users, allowing people to better enjoy a selfdetermined life. Technological advancements are often regarded as crucial means to support an ageing society. The combination of computer-enabled appliances just around the corner on the one hand, plus the need for assistive technology on the the other hand, somewhat naturally resulted in research which is attributed to the field of ambient assistive living (AAL) [17]. The field of AAL is closely related and largely overlapping with the field of ambient intelligence (AmI) [2]. While AAL is more application-driven, AmI takes a broader view and considers use cases beyond assistance for the elderly [10]. AmI may be regarded as more research-driven too, originating by name in a European research initiative. By topic, it can be characterised as the convergence of ubiquitous computing, sensor technology, and artificial intelligence [26]. Technology-driven research in this area is attributed to the third member of this family: smart environments. It is applied to address several challenges, primarily comfort of living and industrial applications, e.g., Industrie 4.0, Smart Factory [19]. Most of the examples in this article refer to smart home applications as we assume readers to be familiar with such environments and because of the currently high commercial visibility of products such as Google Home. But the positioning of the field and the research ques- 
tions stated in this paper apply to the general class of smart environments.

The joint motivation underlying all research fields is to investigate how "smart technology" might improve our private and professional life, and how that technology can be realised. To arrive at our desired goal, several disciplines have to contribute, ranging from technical disciplines involved with sensors and networks to artificial intelligence and human-computer interaction, and beyond [1].

In the remainder of this article we take a closer look onto the research challenges that need to be mastered in order to implement the improvements envisioned by smart environments. First, Section 2 develops a definition of smart environments and positions research in the field with respect to major research directions of artificial intelligence (AI) and human-computer interaction (HCI). In Section 3 we then discuss research questions on a technical level. Finally, the paper ends by a conclusion that reflects chances and risks.

\section{What is being smart?}

Many everyday household devices are advertised as being smart - there is almost no category of devices or appliances for which no "smart" alternative is available. Light switches are acclaimed as being smart because we can access them both in the physical and virtual world, for example by means of a wireless connection from our mobile phone. Starting a dedicated app is sometimes promoted as being easier than literal interaction at the push of a button. From a sales point of view remote controlled devices with little automation capabilities (for example a timer) qualify as smart devices, it seems. As outlined in the previous section, microprocessors with connectivity are becoming ubiquitous in everyday devices, hence technological advancement will turn all devices into smart devices if we would be willing to accept that de-facto definition. For a research field this view would be of little use, though, due to a lack of challenges and research questions. Indeed, already a closer look at simple applications such as the supposedly smart light switch reveals that achieving a more advanced solution that would activate the 'right' light would require to tackle context awareness. Even a technical solution only considering location of user and lamps as a mock-up of context awareness requires a substantial infrastructure (including interoperability of potentially heterogenous components), which is not readily available. This requires us to acknowledge that being smart necessitates mastering situatedness in interaction and reasoning, it needs to blend advancements in sensor and interaction technology with artificial intelligence research.

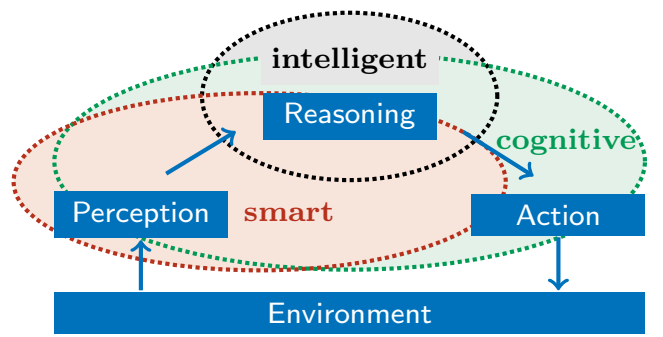

Fig. 1 Coarse architecture of a rational agent according to [25] with focal areas smart, intelligent, and cognitive.

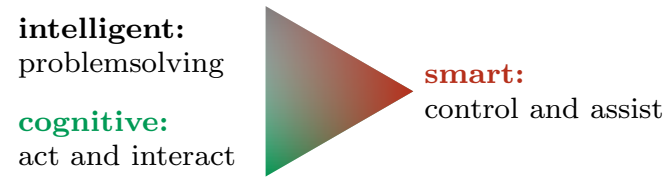

Fig. 2 Contrast of aims for three AI flavours.

\subsection{The Flavours of Artificial Intelligence}

Artificial intelligence is a vast field driven by various motivations. Among the driving forces of AI research, the desire of constructing intelligent machines that support humans has triggered several research questions concerning methodology and engineering techniques. In this constructive branch of AI there are different focal areas that all aim at developing useful systems that gain their usefulness from their ability to perform intelligent decisions or actions autonomously. Several attributes have been introduced to designate these focal areas, prominently intelligent, cognitive, and - most recently - smart. These areas are hard to define in a crisp and discriminatory manner, they blend in various ways - we refer to them as the flavours of AI and characterise them based on a depiction within the classic agent-based architecture of artificial intelligence shown in Fig. 1.

Intelligent systems are often associated with purely symbol-based methods that exhibit sophisticated processing techniques on an abstract level. Even when combined with perception and action, the latter tasks are considered add-ons. By contrast, cognitive systems more closely relate to the idea underlying embodiment in that perception and action are integral parts of the problem. Moreover, cognitive systems often relate to human cognition. As has been argued above, the flavour of smartness was coined by the desire for more advanced sensor-based embedded systems, hence smart systems are closely related to cognitive systems, but rather driven by technological development. Smart systems do not necessarily expose a high level of intelligence in their actions, in particular if their possibilities to act or interact are restricted. The technical nature of smart systems and a realisation possibly unrelated to human cognition motivates coinage of a new term. Spe- 
cial aims pursued by any of the flavours are contrasted in Fig. 2.

We say a system is a smart system whenever it employs sensors to perceive its environment, interprets the perception on an abstract level to yield some non-trivial ("intelligent") decisions beyond simple reactive senseact rules, and is able to interact with the environment or alter it using some actors. In short, smart systems acquire and apply knowledge in order to assist people (cp. [30]). In order to provide a desired service to its users, interaction capabilities are required - these have to reflect the capabilities of the inner system. Even a smart system achieving human-level intelligence in decision-making will not be applied effectively unless interaction with the system allows users to grasp and unleash its functionality.

To make the distinction clearer, let's consider the example of an indoor route guidance system whose task is to guide a person to a specified location. It might have a map, possibly with some level of abstraction like labels of rooms. An intelligent system would use a path planner focussing on how to compute the optimal route (as defined by some optimality criterion such as distance). The result would typically be communicated to the user by a standard user interface such as the display of the route on a smart phone. It may be programmed to recalculate the route based on updates on the position of the user. A cognitive system would possibly use different methods for finding the path such as biologically inspired methods, possibly without an explicit optimality criterion. Most importantly, it would also respond to actions by the user it perceives: When a user was not following the suggested path a cognitive system might actively ask her why she did not follow the route or estimate underlying reasons, eventually adapting to the user's suggestion. By contrast, a smart system would (in addition to a static map) use sensors in the environment to enrich the world model, thus being able to avoid crowded areas. It might also use different interaction methods. Instead of - or in addition to the display on the phone it could activate signals in the environment to guide the user.

If we draw near long-term goals of AI, these differences with respect to the applications will be diminishing. The ultimate goal of all three types of systems demands for 'AI completeness', which implies that if one area had solved the complete puzzle of intelligence, the same solution would be applicable for the other types of systems. Thus, in a distant future the three types of systems would merge into one. However, being still far from this ultimate goal, intelligent, cognitive and smart systems represent three views that may significantly differ in their assumptions, methods, and goals. For ex- ample, the definition of a "good" solution may involve concepts such as rationality, cognitive plausibility, or user satisfaction. The diversity in assumptions, methods and goals is necessary to make progress towards common long-term goals. In addition, the contemporary smart road towards AI completeness promises to open up a way along low-hanging and important application fruits.

\subsection{Smart Environments - an AI Perspective}

In the light of the above classification, we define smart environments as ubiquitous and interactive smart systems that are embedded in the physical environment. Smart environments advance an otherwise passive environment to become an active partner of its users. Setting all technical challenges aside, this leads to a view of two core areas contributing to smart environments: Artificial intelligence for understanding how perception can be linked to appropriate actions and human-machine interaction for understanding how a system can be linked with its users.

\subsection{Role within Artificial Intelligence}

Functional views on smart environments such as the one held by Cook and Das single out the system's ability "to acquire and apply knowledge about the environment and its inhabitants" [11]. This puts classical AI topics such as machine learning, knowledge representation, and reasoning in it's broadest sense into a central position. We may thus regard smart environments to be a demonstrator for AI, showcasing and evaluating methods in context of a real-world application. However, the link between AI and AmI has also been characterised as a chicken-and-egg type of question by Ramos et al. [27], questioning whether AmI would import AI techniques or, conversely, AI would import AmI - the question can analogously be stated for the role of smart environments and AI. The authors claim that realising a smart environment would be a natural next step for AI research that advanced from single-computer intelligent to networked multi-agent systems and is now about to blend in with the environment. This next step would lead to new challenges. If we analyse potential challenges more closely, we identify a central aspect in smart environments research: situatedness. We are involved with problems that require contextual awareness, which means that the situation of users - both physically in their environment as well as cognitively - have to be considered. Context information is not 
available as direct input, but must be derived by sensible interpretation from inputs available. Situatedness also means that the system has to obtain its inputs using sensors, introducing all challenges of handling noisy and uncertain sensor data. In contrast to other AI topics based on sensory input such as mobile robots, smart environments employ a different set of sensors embedded in the environment, for example simple switches detecting door states or ambient light sensors. We need to learn which types of information a specific combination of sensors allows us to reveal, both from the perspective of obtaining required input to context models and from the perspective of privacy. Last but not least, validation of Moore's law provides us with comprehensive yet cheap sensor network installations that enable us to experiment with massive data (exploitation of as well as coping with) in manageable lab-scale environments. While data-driven AI methods receive much attraction these days, we have also take into account whether technically feasible solution will be accepted by users, again taking privacy concerns into account.

\subsection{Role within Human-Machine Interaction}

Situatedness also offers means to exploit the environment. From the viewpoint of human-machine interaction, smart environments present a multitude of new options for interacting with users and offering new services. While classical user interfaces rely on explicit input from users, smart environments can offer proactive services. Output devices are not any more restricted to 2D screens or paper, but can be integrated unobtrusively into household devices. Even more, the environment itself or objects therein equipped with actuators can become communication devices. A coffee machine automatically turning on may nudge employers to have a break, for example.

Technology is already supporting eldercare with automatic pill dispensers, mats or motions sensors to detect falls, or automatically cleaning toilets. The availability of such specific devices leads people to extrapolate future options of situation-based reminding, recognition of the health status and entertainment by smart environments or robots (or combinations of both).

An important aspect of human-machine interaction that is often overlooked by AI researchers is that people are not machines. Neither are we logical inference machines nor do we employ rules of rational decisionmaking $[28,3]$. On the other hand, people bring a hoard of common-sense knowledge and personal memories, allowing them to interpret the world effectively even with limited sensory information. AI researchers know that the tasks that are easiest for humans are the most difficult for computers (and in some cases vice versa). But users do not. If a machine has worked well in a number of cases, users assume that it works well in all the circumstances that they consider similar. The tragic accident of the Tesla car using the autopilot functionality illustrates this fact [6]. Even if the manual tells the user to supervise the system, people still feel safe if the machine has worked well before and since machines and humans work in such different ways, people cannot foresee when the machine will fail. Thus, it may not be necessary (or possible) that smart environments work under every circumstance, but they should be able to communicate to the user when a situation arises in which they stop working. On possible solution could be to introduce system explainability, allowing a system actively to counter-act misconceptions.

Such considerations are not just an add-on to AI systems, they must be considered in the development of the AI techniques themselves.

\section{How to be smart}

Our definition of smart environments positions the field on the boundary of AI and HCI. As we have argued above, smart environments stimulate a unique set of research questions which cannot be answered by importing answers from related fields. In this section, we review research questions central to smart environments. On the one hand, some technological challenges are specific to smart environments, on the other hand existing research questions from AI and HCI develop new potential in the light of this new technological context.

Sensor Systems. Sensors provide the basis for perceiving the state of the working environment. Not only due to the wide range of applications, there is no standard set of sensors and gold standard of their installation (e.g., considering coverage required), but a plethora of options is considered. Further investigations are required to identify the best practice of sensor installation per application. In contrast to mobile robots which often employ rather few but sophisticated sensing devices, smart environments are often based on rather cheap but numerous sensors. It is yet to be clarified which combination of sensors allows which pieces of information to be revealed best. The use of several sensors, many of which provide limited information (for example, a door contact switch) requires them to be connected in sensor networks, which allows more meaningful information to be obtained than from a single sensor alone [24, 4]. Also, this puts sensor fusion up on the agenda of research objectives in the field. Although not in the focus 
of this article, we shall not completely disregard technical challenges of system integration that arise when interconnecting a wide range of different devices.

Robustness and Interoperability. When relying on a complex technical system to provide input for an application one has to take issues arising from failures or system updates into consideration. Sensors may fail or may get updated, potentially affecting characteristics of the data quality (for example, sensor models may become obsolete); coverage or even modality of perception may change. The requirement to implement smart behaviour without relying on the presence of a specific type of sensor at a specific location leads to the need for a semantic interface level (for an overview see [9]) and the ability to identify in an autonomous manner which sensors are available and how information they provide can be exploited.

Service Robots vs. Smart Environments. Different research directions and technologies contest one another to improve our everyday life. If an environment were populated by truly universal, sophisticated service robots, would there be a need for additional smart environment installations? We have to identify the advantages and trade-offs of distinct technologies with respect to effectiveness of the service, economic costs, and user satisfaction. Often, service robots are considered to be part of smart environments [12], yet the balance of technologies has to be investigated.

User and Situation Modeling. Smart systems are expected to understand real-world contexts and act appropriately in a wide range of situations. A basic prerequesite is an understanding of people, their habits and expectations. This is challenging on a general level, but even more so on an individual level. It includes the identification of users, possibly some categorisation into user groups or even individual preferences. Especially systems in the home, but also in public or work places demand a high level of privacy, which often disqualifies methods that rely on large data sets. At least for adaptations to specific users, systems will have to cope with sparse data.

There are also specific demands on knowledge representation. A user action is not a planning operator and human habits are not PDDL plans. We have to take cognitive principles into account, even when interpreting elementary actions such as human navigation [13]. People show stereotypical behaviour, but with slight variations. Capturing such variability while still being able to discriminate situations raises new questions for representation and learning.
Smart environments thus have to cope with a complex interaction of the environment, users and whole situations. In addition to the challenges just mentioned for the representation of each of these components, new concepts of modularity may be necessary to be able to represent complex relations and interdependency, while at the same time ensuring computational feasibility.

Adaptability. For user models as well as the system behaviour the ability to adapt is usually regarded as a basic requirement for smart environments. But in this case, we have (at least) two adaptable systems interacting: the user and the machine. People are known to adapt much better than machines, so the question is whether systems should indeed change or rather come with a well-usable interface to be configurable by people. Norman [23] argues that the most important feature of modern machines is their predictability. On the other hand, people are known to accept default values even though they have other demands [20,22]. Similarly, while in webdesign the "design for all" paradigm is regarded as the gold standard, there is research on adaptive user interfaces [8]. It is likely that the solution depends on the specific task and context and that combinations of explicit configuration and automatic adaptation may be most appropriate. Adaptability in the sense of explicit programming is discussed further below.

Explainability. To make smart environments predictable in the sense of Norman's criteria [23], any non-trivial system decision must be explainable to the user. This is particularly important for smart environments that feature adaptability. When having a garden party in the evening, a user of a smart home needs to know whether the system recognised the party or mis-classifies going into the garden as an action of leaving the house, thus closing the blinds an locking up the house. Explainability of complex AI systems has already been recognised as an issue for acceptability, documented for example by DARPA's current XAI ${ }^{1}$ initiative. Unlike XAI's program aiming for an add-on explanation component to an otherwise black box learning-based system, explanations in context of knowledge-based systems have been around since the first golden years of AI, for example using qualitative process descriptions [14].

Communication. Configuration, but also everyday interaction, raises the question of appropriate communication between a person and a machine and vice versa. The proliferation of mobile and ubiquitous devices has

\footnotetext{
1 see http://www.darpa.mil/program/ explainable-artificial-intelligence
} 
led to new concepts of user interfaces, spanning a spectrum from explicit (pressing a button) to more implicit (gesturing). Smart environments offer new possibilities of system output. Instead of displaying information explicitly on a screen or speaking it out loud, more subtle information such as controlling light (for example to draw the user's attention to some device) or acting on household devices (like switching off the stove) are possible. In both directions, it becomes less obvious whether any communication is intended (Is a user required to react on the change of a light? Is the user trying to control a machine by gesture or is she just scratching her head?). It also raises ethical question of paternalism by the machine. Should any machine have the right to set the light or control household devices without explicit permission?

These questions become even more intricate with systems that try to identify emotions and act accordingly. Even with a perfect recognition, it is questionable whether people want or need machines to know about something as personal as their feelings. But in the light of imperfect recognition, wrong machine behaviour could even endanger users, for example when the machine misclassifies a hysteric fit as laughter.

Programming. A special form of communication is the adaptation of a smart environment in long-term use to new users and tasks as well as changing requirements. Again there is a wide spectrum from classical, explicit methods such as general programming or simplified domain-specific programming languages $[15$, for example], all the way to more implicit natural language instructions and autonomous learning from observation. The more autonomous forms of long-term adjustment suffer again from limited user control and the mutual adaptation of people and machines as discussed above.

Open-world Problem Solving. AI usually assumes rationalistic models for problem solving, be it logic or decision theory. People are well known not act according to these notions of rationality $[18,16]$, and for a good reason: Realistic environments are not just uncertain in the sense of probability theory, they contain unmodelled and/or unknown values and their complexity inhibits the development of reliable (probabilistic) models [16]. Machines that are to work in human environments and with humans must show the same resilience to unknown data and incomplete models. Besides, the behaviour of the machine should be understandable to users.

Thus, for AI to comply with user needs it has to find a balance between the classical "rationalistic" view of problems to a more HCI-driven "design" approach [29].
Evaluation and Methodology. AI methods are usually tested on objective criteria such as recognition rates, optimality or runtime. Systems whose only purpose is to serve humans must be evaluated according to user needs and preferences. This implies user tests as the basic evaluation mechanism. User tests are usually more laborious than typical computer-centred tests, and the test data is limited by the tasks and number of users. Another complication is the definition of test criteria. The most straightforward way is to ask the opinion of users, possibly on a scale to simplify analysis. However, it has been shown in different domains that users do not know what they need [20,22]. Especially for future technologies such as smart home appliances, users are known for not being able to assess their own needs and possible usage habits. Therefore, behavioural data is always preferable. An practical and accepted set of evaluation criteria has yet to be developed though.

Ethics, Freedom, Privacy. While design and technical implementation of smart environments are subject to research in HCI, AI, and further disciplines of computer science, the implementation also raises many ethical and legal questions. This includes the right of and need for privacy, the right or necessity of systems to be understandable for users, and basic questions of whether a machine can or should replace contact between people.

By employing a system that provides assistance with the ability to control elements in an environment automatically we delegate self-determined decisions to an automated unit, thus potentially risking personal freedom [7]. Therefore we have to analyse carefully whether users would gain or loose control in a final account.

If proactive systems are deployed in direct and continuous contact to humans, motivations underlying their decisions should be clearly communicated. That is, the system must be self-explanatory, for example by explaining its decisions to the user.

Moreover, end-user systems pursue economic goals, to the moment of sale and possible beyond. Will a system only act to maximise user satisfaction or is there bias towards an explicit or implicit economic motivation by the vendor? From a business revenue perspective, Accenture has introduced the term "silent commerce" (see critical discussion in $[7,5]$ ) which comprises several means of situated purchase scenarios, including Amazon's dash button. If a smart refrigerator actually implements the widely used example of automatically placing and order when running short of milk, would it consider the local farm shop or order from a major supermarket which maybe proved to provide an easyto-integrate software API or is otherwise affiliated with the provider of the system? At this point, explainabil- 
ity of an AI system should no longer be considered a desiderata, but a legal requirement.

\section{Conclusions}

Smart environments are an active area of research and constitute a promising source of innovation, embedded in research in the fields of artificial intelligence and human-computer interaction. The systems conceived in this field are situated, they gain their usefulness by contextual awareness obtained by interpreting sensor data. Being smart does not require a system to present the full catalog of intelligence, but a restricted set of wellchosen AI and HCI methods can empower valuable applications. Several technological challenges arising with realisations of smart environments have to be solved, too. The most prevailing perspective on smart environments is however that of the users: aside from the HCI perspective on situated interaction, a system penetrating into people's daily life bears dangers for individuals and societies at whole. Factoring aspects of sociology, ethics, and concerns of privacy into research and development in AI and HCI have never been as important before.

\section{Acknowledgments}

Diedrich Wolter acknowledges financial support by Technologieallianz Oberfranken (TAO).

\section{References}

1. Emile Aarts and Boris de Ruyte. New research perspectives on ambient intelligence. Journal of Ambient Intelligence and Smart Environments, 1, 2009.

2. Giovanni Acampora, Diane J. Cook, Parisa Rashidi, and Athanasios V. Vasilakos. A survey on ambient intelligence in health care. Proceedings of the IEEE, 101:24702494, December 2013.

3. Dan Ariely. Predictably Irrational: The Hidden Forces That Shape Our Decisions. Harper, New York, NY, 2008.

4. Luca Benini, Elisabetta Farella, and Carlotta Guiducci. Sensor networks for ambient intelligence. Microelectronics Journal, 37(12):1639-1649, December 2006.

5. Jürgen Bohn, Vlad Coroamă, Marc Langheinrich, Friedemann Mattern, and Michael Rohs. Social, economic, and ethical implications of ambient intelligence and ubiquitous computing. In Ambient Intelligence, pages 5-29. Springer, 2005.

6. Neal E. Boudette. Tesla's self-driving system cleared in deadly crash. The New York Times, 19 January 2017.

7. Philip Brey. Freedom and privacy in ambient intelligence. Ethics and Information Technology, 7(3):157-166, 2006.

8. Dermot Browne, Peter Totterdell, and Mike Norman, editors. Adaptive User Interfaces. Academic Press Ltd., London, UK, 1990.
9. Michael Compton, Cory A. Henson, Holger Neuhaus, Laurent Lefort, and Amit P. Sheth. A survey of the semantic specification of sensors. In Proceedings of the 2nd International Workshop on Semantic Sensor Networks ( SSN09), collocated with the 8th International Semantic Web Conference ( ISWC-2009), Washington DC, USA, October 26, 2009., pages 17-32, 2009.

10. Diane J. Cook, Juan C. Augusto, and Vikramaditya R. Jakkula. Ambient intelligence: Technologies, applications, and opportunities. Pervasive and Mobile Computing, 5:277-298, August 2009.

11. Diane J. Cook and Sajal K. Das. How smart are our environments? An updated look at the state of the art. Pervasive and Mobile Computing, 3:53-73, 2007.

12. S. Coradeschi and A. Saffiotti. Symbiotic robotic systems: Humans, robots, and smart environments. IEEE Intelligent Systems, 21:82-84, 2006.

13. Hannah M. Dee and David C. Hogg. Navigational strategies in behaviour modelling. Artificial Intelligence, 173:329-342, 2009.

14. Kenneth D. Forbus. Qualitative process theory. Artificial Intelligence, 24(1-3):85-168, December 1984.

15. Manuel García-Herranz, Pablo Haya, and Xavier Alamán. Towards a ubiquitous end-user programming system for smart spaces. Journal of Universal Computer Science, 16:1633-1649, 2010.

16. Gerd Gigerenzer and Wolfgang Gaissmaier. Heuristic decision making. Annual Review of Psychology, 62:451-482, 2011.

17. Christina Jaschinski and Somaya Ben Allouch. An extended view on benefits and barriers of ambient assisted living solutions. International Journal on Advances in Life Sciences, 7, 2015.

18. Daniel Kahneman. Thinking, Fast and Slow. Farrar, Straus and Giroux, New York City (NY), USA, 2011.

19. Dominik Lucke, Carmen Constantinescu, and Engelbert Westkämper. Smart factory - a step towards the next generation of manufacturing. In M. Mitsuishi, K. Ueda, and F. Kimura, editors, Manufacturing Systems and Technologies for the New Frontier, Proceedings of the 41st CIRP Conference on Manufacturing Systems, May 26-28, 2008, Tokyo, Japan. Springer, 2008.

20. Kat Matfield. Bridging the gap between actual and reported behavior. http://www.uxbooth.com/articles/bridging-the-gapbetween-actual-and-reported-behavior/, May 2015.

21. Gordon E. Moore. Cramming more components onto integrated circuits. Electronics, 38:114-117, 1965.

22. Richard E. Nisbett and Timothy DeCamp Wilson. Telling more than we can know: Verbal reports on mental processes. Psychological Review, 84(3), 1977.

23. Don Norman. The Design of Future Things. Basic Books, 2007.

24. Eric Pauwels, Albert Ali Salah, and Romain Tavenard. Sensor networks for ambient intelligence. In Proceedings of IEEE Workshop on Multimedia Signal Processing, Chania, Greece, October 2007.

25. Stuart Russell and Peter Norvig. Artificial Intelligence: A Modern Approach. Prentice Hall Press, Upper Saddle River, NJ, USA, 3rd edition, 2009.

26. Fariba Sadri. Ambient intelligence: A survey. ACM Computing Surveys, 43:Article 36, October 2011.

27. Daniel Shapiro, Juan Carlos Augusto, and Carlos Ramos. Ambient intelligence - the next step for artificial intelligence. IEEE Intelligent Systems, 23(2):15-18, 2008. guest editors' introduction. 
28. Amos Tversky and Eldar Shafir. The disjunction effect in choice under uncertainty. Psychological Science, 3(5):305-310, 1992.

29. Terry Winograd. Shifting viewpoints: Artificial intelligence and human-computer interaction. Artificial Intelligence, 170(18):1256-1258, 2006.

30. G. Michael Youngblood, Edwin O. Heierman, Lawrence B. Holder, and Diane J. Cook. Automation intelligence for the smart environment. In Proceedings of the International Joint Conference on Artificial Intelligence, 2005. 